\title{
$S d$ - Topology over the theory of a Model
}

\author{
Soham Dasgupta \\ Ex. PGT (Math) at Kendriya Vidyalaya Ordnance Factory, Dumdum, Kolkata, W.B, India
}

\begin{abstract}
Construction of a new topology over the theory of a Model to visualize and discuss some of its interesting properties and results. It's actually creating a bridge between model theory and topology.
\end{abstract}

Keywords: Theory, Sub Theory, Theorem, Kuratowski’s Closer Operator, Topology, Sd-Topology

\section{INTRODUCTION}

The main inspiration of this paper comes from 'Vedas', which is an ancient collection of some fundamental theories and itself a theory. Now the question arises that when and how a collection of theories can itself become a theory? For searching the answer of it I tried to visualize the very basic Model Theoretic and logical topics through the spectacles of a particular topological space defined over the theories. This non standard effort leads to some interesting properties and results.

\section{DEFINITION}

If $\mathcal{U}$ is a model for the language $\mathscr{L}$, the theory of $\mathcal{U}$ is denoted by $\mathbf{T h} \mathcal{U}$, is defined to be the set of all sentences of $\mathscr{L}$ (i.e formulas with no free variables) which are true in $\mathcal{U}$.

So Th $\mathcal{U}=\{\sigma$ of $\mathscr{L}: \mathcal{U} \vDash \sigma\}$

For example if $\mathscr{L}=\{\langle,+, \cdot, 0,1\}$ then $\mathbf{T h} \mathbb{R}$ is famous as the name Real Analysis. Similarly over the same language $\mathbf{T h} \mathbb{Z}$ is known as number theory again for $\mathscr{L}=\{+, \cdot, 0,1\} \mathbf{T h} \mathbb{C}$ is renowned as complex analysis.

[ Note: here we denote the models for a given language as italic notations as the universe of the model i.e $\mathbb{R}=\langle\mathbb{R}, I>$ where $I$ is the respective interpretation function, also further we indicate the cardinality of a model as the cardinality of its universe i.e card $\mathbb{R}$ means $\operatorname{card} \mathbb{R}=c]$

\section{Construction of $S d$-topology on Th $\mathcal{U}$ (When card $\left.\mathcal{U} \geq \boldsymbol{\aleph}_{0}\right)$ :}

Lets define a mapping $k: \mathrm{P}(\mathbf{T h} \boldsymbol{U}) \rightarrow \mathrm{P}(\mathbf{T h} \mathcal{U})$ as

$k(\mathrm{~A})=\mathrm{A}$ if card $\mathrm{A}<\operatorname{card} \boldsymbol{U} \quad$ [ where A is subset (i.e sub theory) of $\mathbf{T h} \boldsymbol{U}$ ]

$k(\mathrm{~A})=\mathbf{T h} \boldsymbol{U}$ if card $\mathrm{A} \geq \operatorname{card} \boldsymbol{U}$

It's easy to verify that $\mathrm{k}$ is a Kuratowski's Closer Operator

since $k(\varnothing)=\varnothing, \mathrm{A} \subseteq k(\mathrm{~A}) \& k(k(\mathrm{~A}))=k(\mathrm{~A}) ; \forall \mathrm{A} \subseteq \mathbf{T h} \boldsymbol{U}$ and $k(\mathrm{~A} \mathrm{U} \mathrm{B})=k(\mathrm{~A}) \mathrm{U} k(\mathrm{~B}) ; \forall \mathrm{A}, \mathrm{B} \subseteq \mathbf{T h} \boldsymbol{U}$

LEMMA: Let $k$ be a Kuratowski's Closer operator on a set $X$. Then there is a unique topology $\tau$ on $X$ such that $\mathrm{k}(\mathrm{A})=\overline{\mathrm{A}}$; in $(\mathrm{X}, \tau) \forall \mathrm{A} \subseteq \mathrm{X}$ where $\overline{\mathrm{A}}$ is the closer of $\mathrm{A}$.

Proof: Let $\sum=\{\mathrm{k}(\mathrm{A}): \mathrm{A} \subseteq \mathrm{X}\}$ since $\mathrm{k}(\mathrm{k}(\mathrm{A}))=\mathrm{k}(\mathrm{A}), \forall \mathrm{A} \subseteq \mathrm{X}$

so we can treat $\sum=\{\mathrm{A} \subseteq \mathrm{X}: \mathrm{k}(\mathrm{A})=\mathrm{A}\}$, Obviously $\emptyset \in \sum$ and $\mathrm{X} \in \sum$

Again if $\mathrm{A}, \mathrm{B} \in \sum$ then as $\mathrm{k}(\mathrm{A} \mathrm{U} \mathrm{B})=\mathrm{k}(\mathrm{A}) \mathrm{U} \mathrm{k}(\mathrm{B})=\mathrm{A} U \mathrm{~B}$, so $\mathrm{AUB} \in \sum$ thus $\sum$ is closed under finite union. Again if $A_{i} \in \sum$ then as $\cap_{i} A_{i} \subseteq A_{i}$ so by the property of $k$,

$\mathrm{k}\left(\cap_{\mathrm{i}} \mathrm{A}_{\mathrm{i}}\right) \subseteq \mathrm{k}\left(\mathrm{A}_{\mathrm{i}}\right)=\mathrm{A}_{\mathrm{i}}$ therefore $\bigcap_{\mathrm{i}} \mathrm{A}_{\mathrm{i}} \subseteq \mathrm{k}\left(\cap_{\mathrm{i}} \mathrm{A}_{\mathrm{i}}\right) \subseteq \bigcap_{\mathrm{i}} \mathrm{A}_{\mathrm{i}}$ and $\mathrm{k}\left(\cap_{\mathrm{i}} \mathrm{A}_{\mathrm{i}}\right)=\bigcap_{\mathrm{i}} \mathrm{A}_{\mathrm{i}}$ thus $\cap_{i} A_{i} \in \sum$ so it's also closed under arbitrary intersection. Therefore $\tau$ is defined as $\tau=\left\{\mathrm{V}: \mathrm{X}-\mathrm{V} \in \sum\right\}$, surly a topology on $\mathrm{X}$ with $\sum$ is the set of all closed sets in $(\mathrm{X}, \tau)$. Now $\bar{A} \subseteq k(A)$ since $k(A)$ is a closed set containing $A$. Again $\bar{A} \subseteq k(\bar{A}) \subseteq k(k(A))=k(A)$. But $\mathrm{k}(\mathrm{A}) \subseteq \mathrm{k}(\overline{\mathrm{A}})$ so we get $\mathrm{k}(\mathrm{A})=\overline{\mathrm{A}}$. 
For uniqueness if $\tau_{1}$ and $\tau_{2}$ are two topologies on $\mathrm{X}$ such that $\mathrm{Cl} \tau_{1}(\mathrm{~A})=\mathrm{Cl} \tau_{2}(\mathrm{~A}), \forall \mathrm{A} \subseteq \mathrm{X}$. Then $\left\{\mathrm{Cl} \tau_{1}(\mathrm{~A}): \mathrm{A} \subseteq \mathrm{X}\right\}=\left\{\mathrm{Cl} \tau_{2}(\mathrm{~A}): \mathrm{A} \subseteq \mathrm{X}\right\}$ so $\tau_{1}=\tau_{2}\left(\right.$ Where $\mathrm{Cl} \tau_{1}(\mathrm{~A})=\overline{\mathrm{A}}$ in $\left.\left(\mathrm{X}, \tau_{1}\right)\right)$ ( Q.E.D)

Now with the help of this lemma and the Kuratowski's Closer Operator ' $k$ ' what we had defined earlier , we can construct a unique topology over $\mathbf{T h} \mathcal{U}$. Where $k(\mathrm{~A})=\overline{\mathrm{A}}, \forall \mathrm{A} \subseteq \mathbf{T h} \mathcal{U}$. We called that topology is the $\boldsymbol{S d}$-topology on Th $\boldsymbol{U}$. We are denoting that topology further as $\tau_{\mathrm{Sd}}$. Another thing to note that if $\boldsymbol{c a r d} \boldsymbol{\boldsymbol { U }}<\boldsymbol{\aleph}_{\mathbf{0}}$ or $\operatorname{card}(\mathrm{Th} \boldsymbol{U})<$ card $(\mathcal{U})$ then $\left(T h \mathcal{U}, \tau_{S d}\right)$ will be very boring and elementary so in general we consider $\left(T h \mathcal{U}, \tau_{S d}\right)$ with card $\boldsymbol{U} \geq \boldsymbol{\aleph}_{\mathbf{0}}$ and $\operatorname{card}(\operatorname{Th} \boldsymbol{U}) \geq \operatorname{card}(\mathcal{U})$.

\section{Some Properties of the topological space $\left(\mathrm{Th} \mathcal{U}, \tau_{\mathrm{Sd}}\right):$}

1) In $\left(T h, \tau_{S d}\right)$ any Sub Theory ( i.e non trivial subsets of a theory) containing equal or more sentences than the cardinality of the universe of the respective model, then it's closer is itself the theory. In other words we can say that in $\left(T h \mathcal{U}, \tau_{S d}\right)$ if $A \subseteq \boldsymbol{T h} \mathcal{U}$ and $\operatorname{card}(A) \geq \operatorname{card}(\mathcal{U})$ then $A$ is dense in $\left(T h \mathcal{U}, \tau_{S d}\right)$.

Proof: Its trivial to check that as the operator defined as $k(\mathrm{~A})=\mathbf{T h} \mathcal{U}$ if card $\mathrm{A} \geq$ card $\mathcal{U}$. Also as in $\left(T h, \tau_{S d}\right), \mathrm{k}(\mathrm{A})=$ $\overline{\mathrm{A}}, \forall \mathrm{A} \subseteq \mathbf{T h} \boldsymbol{U}$. So whenever card $\mathrm{A} \geq$ card $\boldsymbol{U}$ then $\overline{\mathrm{A}}=\boldsymbol{T h} \boldsymbol{U}$. (Q.E.D)

2) In $\left(T h, \tau_{S d}\right)$ any Sub Theory A with $\operatorname{card}(A)<\operatorname{card}(\mathcal{U})$ is closed. Also vise-versa.

3) If $\left(T h \mathcal{U}, \tau_{S d 1}\right)$ and $\left(T h \sum, \tau_{S d 2}\right)$ are two $S d$-topological spaces with card $\mathcal{U}=$ card $\sum$ then any bijection (if exists) between $T h \mathcal{U}$ and $T h \sum$ is a Homeomorphism.

Proof: Let f: $T h \mathcal{U} \rightarrow T h \sum$ be a bijection, then $\operatorname{card}(\operatorname{Th} \mathcal{U})=\operatorname{card}\left(\operatorname{Th} \sum\right)$. Now if A is any proper closed set in $\left(T h \sum\right.$, $\left.\tau_{S d 2}\right)$ then $\operatorname{card}(\mathrm{A})<\operatorname{card}\left(\sum\right)$. Since bijection preserves cardinality then $\operatorname{card}\left(\mathrm{f}^{-1}(\mathrm{~A})\right)<\operatorname{card}\left(\sum\right)=\operatorname{card}(\mathcal{U})(a s$ given). So $\mathrm{f}^{-1}(\mathrm{~A})$ is also closed in $\left(T h \mathcal{U}, \tau_{S d l}\right)$. Therefore $\mathrm{f}$ is continuous. Again $\mathrm{f}$ is also a closed map between the spaces. So $\mathrm{f}$ is a homeomorphism. (Q.E.D)

4) If $\operatorname{card}(\mathrm{Th} \mathcal{U}) \geq \operatorname{card}(\mathcal{U})$ then $\left(T h \mathcal{U}, \tau_{S d}\right)$ is $T_{1}$ but not $T_{2}$;

Proof:T $T_{1}$ because, if $\boldsymbol{\sigma}_{\mathbf{1}}, \boldsymbol{\sigma}_{\mathbf{2}} \in T h \mathcal{U}$ and as card $(\boldsymbol{U})$ and card(Th $\left.\mathcal{U}\right)$ are infinite so $T h \boldsymbol{U}-\left\{\sigma_{1}\right\}$ and $T h \mathcal{U}-\left\{\sigma_{1}\right\}$ are open sets. Its not $T_{2}$ because $\left(T h, \tau_{S d}\right)$ has no proper pair of disjoint open sets.

[ Note: if $\operatorname{card}(\operatorname{Th} \boldsymbol{U})<\operatorname{card}(\boldsymbol{U})$, then $\left(T h \mathcal{U}, \tau_{S d}\right)$ is a discreet space, similarly when card(Th $\left.\boldsymbol{u}\right)$ is infinite and card $\mathcal{U}=\aleph_{0}$ then $\left(T h \mathcal{U}, \tau_{S d}\right)$ is cofinite space, again if $\operatorname{card} \boldsymbol{U}=\aleph_{1}$ then it's a cocountable space]

5) $\left(T h \mathcal{U}, \tau_{S d}\right)$ spaces are connected when $\operatorname{card}(\operatorname{Th} \boldsymbol{U}) \geq \operatorname{card}(\mathcal{U})$

6) In general $\tau_{S d}-\{\varnothing\}$ is an ultra filter in $\left(T h \mathcal{U}, \tau_{S d}\right)$

DEFINITION: A sentence $\sigma \in T h \mathcal{U}$ is said to be a Theorem iff there exist a convergent sequence of different sentences $\left\{\sigma_{\mathrm{n}}\right\}_{\mathrm{n} \in \mathrm{N}}\left(\right.$ i.e steps) in $T h \mathcal{U}$, which converges to $\sigma$ in $\left(T h \mathcal{U}, \tau_{S d}\right)$. Then the set $\left\{\sigma_{\mathrm{n}}: \mathrm{n} \in \mathbb{N}\right\} \mathrm{U}$

$\{\sigma\}$ is called a proof.

Basically as we know that the last line of a proof is called a Theorem.

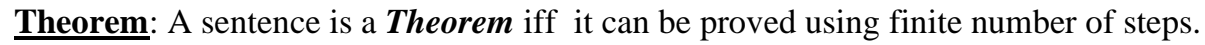

To proof this theorem using finite steps we have to discuss some lemmas in $\left(T h \mathcal{U}, \tau_{S d}\right)$ 
Vol. 7, Issue 12, December 2020

DOI 10.17148/IARJSET.2020.71209

LEMMA 1: In $\left(T h \mathcal{U}, \tau_{S d}\right)$ if card $\boldsymbol{U}=\boldsymbol{\aleph}_{\mathbf{0}}$ a sequence is convergent iff its semi-constant. ( i.e exactly one term of the sequence repeated infinitely many times)

Proof: In $\left(\mathrm{Th} \boldsymbol{U}, \tau_{\mathrm{Sd}}\right)$ if card $\boldsymbol{U}=\boldsymbol{\aleph}_{\mathbf{0}}$ the space is a cofinite space and the above lemma is evident there also that sequence converges to that particular term(point) always. We can find this lemma in almost all general topology book.

LEMMA 2: In $\left(\mathrm{Th} \boldsymbol{u}, \tau_{\mathrm{Sd}}\right)$ if card $\boldsymbol{U}>\boldsymbol{\kappa}_{0}$ a sequence is convergent iff its eventually constant. Proof: Let $\left\{\sigma_{\mathrm{n}}\right\}_{\mathrm{n} \in \mathrm{N}}$ be a non (eventually) constant sequence in $\left(\mathrm{Th} \boldsymbol{u}, \tau_{\mathrm{Sd}}\right)$ converges to $\sigma \in \mathrm{Th} \boldsymbol{u}$. So $\sigma \notin\left\{\sigma_{\mathrm{n}}: \forall \mathrm{n} \in \mathbb{N}\right\}$ but the set $\left\{\sigma_{\mathrm{n}}: \mathrm{n} \in \mathbb{N}\right\}$ is countable, so its closed in (Thu, $\left.\tau_{\mathrm{Sd}}\right)$.

Then Thu- $\left\{\sigma_{\mathrm{n}}: n \in \mathbb{N}\right\}$ is a neighbourhood of $\sigma$ which contains no $\sigma_{\mathrm{n}}$, which is a contradiction.

The converse of the lemma is obvious.

Proof of the theorem: If ( $\left.\mathrm{Th} \boldsymbol{U}, \tau_{\mathrm{Sd}}\right)$ is with card $\boldsymbol{u}=\boldsymbol{\aleph}_{\mathbf{0}}$ then from the lemma 1 we can see that there any sequence is convergent iff its semi-constant. But as the definition of Theorem its proof cannot repeat any of its sentences. Thus for this case the proof should be finite. The converse is also true as by the lemma1. Now if (Th $\left.\boldsymbol{U}, \tau_{\mathrm{Sd}}\right)$ with card $\boldsymbol{U}>\boldsymbol{\aleph}_{\mathbf{0}}$ then by lemma 2 the proof of any theorem should be an eventually constant sequence of sentences. With the same logic as before the proof should be finite. Again by lemma 2 its converse is also true. (Q.E.D)

\section{REFFERENCES}

1. General Topology by J.F Kelly, Springer

2. Introduction to General Topology by K.D.Joshi, New Age Publisher

3. What is Mathematical Logic ? by J.N.Crossley et.al, Dover Publication

4. Fundamentals of Model Theory by Willium Weiss and Cherie D’Mello ( University of Toronto) 